\title{
Nanofluidics is on the rise
}

\author{
Materials discovery and developments in nanofabrication and our understanding of transport at the nanoscale are \\ supporting the rapid development of nanofluidics and accelerating its technological translation.
}

$\Lambda \mathrm{b}_{\mathrm{b}}^{\mathrm{t}}$ $\mathrm{t}$ the nanometre scale, materials behave very differently from their bulk counterparts. This is also true for water and fluids confined within nanostructures. Indeed, nanofluidics the study of water and ion transport at the nanometre scale - has revealed that physical phenomena occur at the nanoscale that cannot be described within the continuum laws of hydrodynamics of bulk fluids. This is related to forces and processes (such as surface charge and steric forces) that can be considered in terms of boundary conditions when dealing with flows at the micrometre and larger scales, yet become quite important when the confinement size goes down to the nanoscale. The high surface-to-volume ratio, non-negligible surface charge and overlapping electric double layer that characterize nanochannels and nanopores lead to non-linear transport mechanisms, fast flows and asymmetric ion transport ${ }^{1-3}$. These findings prompted the development of new theoretical descriptions, experimental approaches and materials for nanofluidics. Moreover, researchers realized that these unusual phenomena could be harnessed in nanofluidic devices for applications, particularly those related to membrane science, energy harvesting and DNA sequencing ${ }^{4-6}$.

The lack of robust methods to fabricate nanofluidic materials with well-defined channel and pore geometries has been the major limiting factor hindering the development of this field. Notwithstanding, several achievements have contributed to the substantial progress of nanofluidics over the past decade, as discussed in a Comment in this issue by Lydéric Bocquet. The most significant were the development of new nanomaterials and nanofabrication techniques, which made the production of nanofluidic devices more reliable, and the technology itself more appealing to an increased community of researchers. With regard to materials, layered two-dimensional materials made from carbon, hexagonal boron nitride, $\mathrm{MoS}_{2}$, MXenes and even clay have been crucial for advancing our understanding of fluid and ion behaviour at such small scales. The interlayer spaces of these materials form long two-dimensional nanoscale channels for water and ions,

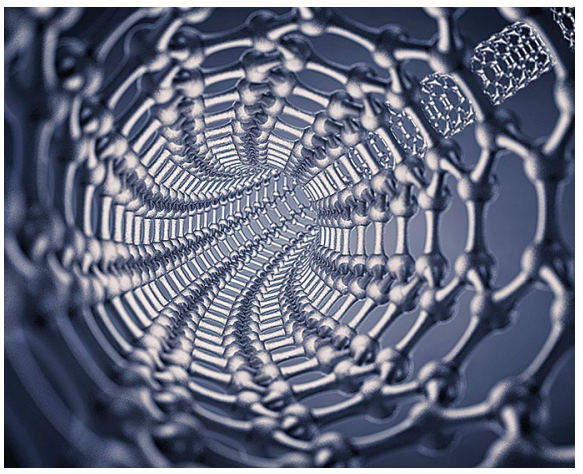

Illustration of the view inside a graphene nanotube, a common nanofluidic device. Credit: Nobeastsofierce Science/Alamy Stock Photo

which can be studied both in experiments and simulations. Moreover, the distance between each layer can be varied, and pores with well-defined sizes and geometries can be fabricated using ion or electron irradiation, both in multi- and single-layered materials. Nanotubes made from some of these materials are also available and can be incorporated with precise configurations into synthetic and lipid membranes, or studied individually. Overall, the improved control over geometrical parameters obtained with these materials enables the fabrication of nanofluidic systems suitable for systematic fundamental investigations and device optimization.

Examples of atypical behaviour in nanoconfined fluids and ions - such as the fast flow of water and ions, selective and asymmetric ion transport, altered properties of liquid water and single-file transport ${ }^{1-3}-$ have now been identified and characterized using the materials and devices mentioned above. While many of these processes still remain unexplained, in-depth knowledge of the performance of nanofluidic systems has been gathered, which supports their use in functional devices. In fact, applications in water filtration and purification ${ }^{4}$, energy harvesting under osmotic driving ${ }^{5}$ and DNA sequencing ${ }^{6}$ have been developed with some success. Nonetheless, as discussed in Bocquet's Comment, several practical challenges lie ahead. Bypassing the selectivity-permeability trade-off, mitigation of fouling and clogging, and fabrication scale-up are some of the hurdles that nanofluidic devices will need to overcome before reaching the industrial setting. Some of these challenges are already being tackled with approaches borrowed by macrofluidics, such as using materials with low water friction to avoid clogging and coatings against fouling. Researchers are also looking into water and ionic channels found in biological organisms. These natural nanofluidic systems have been perfected over millions of years of evolution, combining high selectivity and efficiency with stimulus response and flow control. Filtration membranes incorporating biological pores or pore-forming proteins with performances much superior to those of synthetic alternatives have recently been developed, as described by Yu-Ming $\mathrm{Tu}$ and colleagues in an Article in this issue. Purely synthetic channels with the permeability and selectivity of aquaporins (natural water channels) have also been achieved ${ }^{7}$, supporting the development of such biomimetic devices. However, current hybrid and synthetic nanofluidic systems are still a long way from achieving the remarkable performances and fine control of their biological counterparts.

Despite recent achievements, nanofluidics still has potential to grow both from fundamental and applied perspectives. Materials science, especially its engineering branch, will remain at the core of nanofluidics, but its full potential can only be reached with contributions from other fields of science such as biology, chemistry and physics. Such interdisciplinary collaboration will be particularly relevant for the successful translation of nanofluidics into filtration and energy-harvesting technologies, which could be true game changers.

Published online: 25 February 2020 https://doi.org/10.1038/s41563-020-0633-8

\footnotetext{
References

1. Bocquet, L. \& Charlaix, E. Chem. Soc. Rev. 9, 1073-1095 (2010).

2. Xu, Y. Adv. Mater. 30, 1702419 (2018).

3. Eijkel, J. C. T. \& van den Berg, A. Microfluid. Nanofluidics 1 , 249-267 (2005)

4. Liu, G., Jin, W. \& Xu, N. Chem. Soc. Rev. 44, 5016-5030 (2015).

5. Siria, A. et al. Nature 494, 455-468 (2013).

. Branton, D. et al. Nat. Biotechnol. 26, 1146-1153 (2008).

. Barboiu, M. Chem. Commun. 52, 5657-5665 (2016).
} 